\title{
Involutive Categories and Monoids, with a GNS-Correspondence
}

\author{
Bart Jacobs
}

Received: 14 October 2010 / Accepted: 10 August 2011 / Published online: 8 September 2011

(C) The Author(s) 2011. This article is published with open access at Springerlink.com

\begin{abstract}
This paper develops the basics of the theory of involutive categories and shows that such categories provide the natural setting in which to describe involutive monoids. It is shown how categories of Eilenberg-Moore algebras of involutive monads are involutive, with conjugation for modules and vector spaces as special case. A part of the so-called Gelfand-Naimark-Segal (GNS) construction is identified as an isomorphism of categories, relating states on involutive monoids and inner products. This correspondence exists in arbritrary involutive symmetric monoidal categories.
\end{abstract}

Keywords Involution · Monoidal category $\cdot$ Inner products ·

Gelfand-Naimark-Segal

\section{Introduction}

In general an involution is a certain endomap $i$ for which $i \circ i$ is the identity. The inverse operation of a group is a special example. But there are also monoids with such an involution, such as for instance the free monoid of lists over some set, with list reversal as involution. Involutions are crucial in the reversibility of quantum computation, notably in the adjoint (conjugate transpose) of an operator.

An involution can also be defined on a category $\mathbf{C}$. It then consists of an endofunctor $\mathbf{C} \rightarrow \mathbf{C}$, which is typically written as $X \mapsto \bar{X}$. It should satisfy $\overline{\bar{X}} \cong X$. Involutive categories occur in the literature, for instance in $[1,7,13]$, but have not been studied very extensively. This paper will develop the basic elements of such a theory of involutive categories. Its main technical contribution is a bijective correspondence

B. Jacobs $(\bowtie)$

Institute for Computing and Information Sciences (iCIS), Radboud University, Nijmegen,

The Netherlands

e-mail: bart@cs.ru.nl 
between states $M \rightarrow I$ on an involutive monoid $M$ and inner products $\bar{M} \otimes M \rightarrow I$, relating fundamental notions in the mathematical modeling of quantum phenomena.

We should note that involutive categories as we understand them here are different from dagger categories - which have an identity-on-objects functor $(-)^{\dagger}: \mathbf{C}^{\mathrm{op}} \rightarrow$ $\mathbf{C}$ with $f^{\dagger \dagger}=f$-and also from $*$-autonomous categories-which have a duality $(-)^{*}: \mathbf{C}^{\text {op }} \rightarrow \mathbf{C}$ given by a dualising object $D$ as in $X^{*}=X \multimap D$. In both these cases one has contravariant functors, whereas involution $\overline{(-)}: \mathbf{C} \rightarrow \mathbf{C}$ is a covariant functor. The relation between involution, dagger and duality for Hilbert spaces is described in [2, Sects. 4.1, 4.2]: each can be defined in terms of the other two.

Involutive categories and involutive monoids are related: just like the notion of a monoid is formulated most generally within a monoidal category (see [24, Ch. VII]), the notion of involutive monoid requires an appropriate notion of involutive monoidal category as context of definition. This is in line with the "microcosm principle", formulated by Baez and Dolan [5], and elaborated in [14-16]: it involves "outer" structure-like monoidal structure $1 \stackrel{I}{\rightarrow} \mathbf{C} \stackrel{\otimes}{\leftarrow} \mathbf{C} \times \mathbf{C}$ on a category $\mathbf{C}$-that enables the definition of "inner" structure-like a monoid $I \stackrel{0}{\rightarrow} M \stackrel{+}{\leftarrow} M \otimes M$ inside $\mathbf{C}$. We briefly illustrate how this connection between involutive monoids and involutive categories arises.

Consider for instance the additive group $\mathbb{Z}$ of integers with minus - as involution. In the category Sets of ordinary sets and functions between them we can describe minus as an ordinary endomap $-: \mathbb{Z} \rightarrow \mathbb{Z}$. The integers form a partially ordered set, so we may wish to consider $\mathbb{Z}$ also as involutive monoid in the category PoSets of partially ordered sets and monotone functions. A problem is that minus reverses the order: $i \leq j \Rightarrow-i \geq-j$, and is thus not a map $\mathbb{Z} \rightarrow \mathbb{Z}$ in PoSets. However, we can describe it as a map $(\mathbb{Z}, \geq) \rightarrow(\mathbb{Z}, \leq)$ in PoSets, using the reversed order ( $\geq$ instead of $\leq$ ) on the integers. This order reversal forms an involution $\overline{(-)}$ : PoSets $\rightarrow$ PoSets on the "outer" category, which allows us to describe the involution "internally" as $-: \overline{\mathbb{Z}} \rightarrow \mathbb{Z}$ in PoSets.

As said, this paper contains the basic steps of the theory of involutive categories. It introduces the category of "self-conjugate" objects, and shows how involutions arise on categories of Eilenberg-Moore algebras of an "involutive" monad. This general construction includes the important example of conjugation on modules and vector spaces, for the multiset monad associated with an involutive semiring. It allows us to describe abstractly an involutive monoid in such categories of algebras. Pre $C^{*}$ algebras (without norm) are such monoids.

Once this setting has been established we consider inner products $\bar{V} \otimes V \rightarrow X$, where the input type handles antilinearity (or conjugate linearity) in the first argument (via involution $\bar{V}$ ) and linearity in the second one. Section 7 discusses such inner products, and abstractly reconstructs part of the famous Gelfand-NaimarkSegal (GNS) construction [3], relating $C^{*}$-algebras and Hilbert spaces, and showing in particular how a state $A \rightarrow \mathbb{C}$ on a $C^{*}$-algebra $A$ gives rise to an inner product $\bar{A} \otimes A \rightarrow \mathbb{C}$. The latter map incorporates the sesquilinearity requirements in its type. Section 7 gives an important part of this GNS construction in the form of an isomorphism of categories (see Theorem 2), involving a non-trivial bijective correspondence between states and inner products, using the language of involutive categories and monoids. 
In what follows we assume familiarity with basic category theory, see for instance $[4,10,24]$.

\section{Involutive Categories}

Definition 1 A category $\mathbf{C}$ will be called involutive if it comes with a 'involution' functor $\mathbf{C} \rightarrow \mathbf{C}$, written as $X \mapsto \bar{X}$, and a natural isomorphism $\iota:$ id $\Rightarrow \overline{\overline{(-)}}$ with components $\iota_{X}: X \stackrel{\cong}{\longrightarrow}$ satisfying:

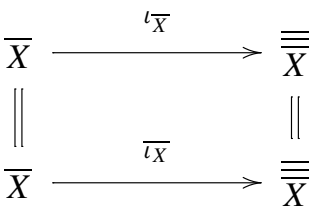

Each category is trivially involutive via the identity functor. This trivial involution is certainly useful. The category PoSets is involutive via order reversal. This applies also to categories of, for instance, distributive lattices or Boolean algebras. The category Cat of (small) categories and functors is involutive, by taking opposites of categories. Next, consider the category $\operatorname{Vect}_{\mathbb{C}}$ of vector spaces over the complex numbers $\mathbb{C}$. It is an involutive category via conjugation. For a vector space $V \in \mathbf{V e c t}_{\mathbb{C}}$ we define $\bar{V} \in$ Vect $_{\mathbb{C}}$ with the same vectors as $V$, but with adapted scalar multiplication $s \cdot \bar{V} v=\bar{s} \cdot V v$, for $s \in \mathbb{C}$ and $v \in V$, where $\bar{s}=a-i b$ is the conjugate of the complex number $s=a+i b \in \mathbb{C}$.

The following is the first of a series of basic observations.

Lemma 1 The involution functor of an involutive category is self-adjoint: $\overline{(-)} \dashv \overline{(-)}$. As a result, involution preserves all limits and colimits that exist in the category.

Proof Obviously there are bijective correspondences:

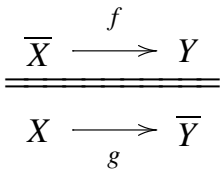

One maps $f$ to $\widehat{f}=\bar{f} \circ \iota_{X}: X \stackrel{\cong}{\longrightarrow} \rightarrow \bar{Y}$ and $g$ to $\widehat{g}=\iota_{Y}^{-1} \circ \bar{g}: \bar{X} \rightarrow \overline{\bar{Y}} \cong Y$. In fact, requirement (1) amounts to the triangular identities for this adjunction.

Definition 2 A functor $F: \mathbf{C} \rightarrow \mathbf{D}$ between two involutive categories is called involutive if it comes with a natural transformation (or distributive law) $v$, with components $F(\bar{X}) \rightarrow \overline{F(X)}$, commuting appropriately with the isomorphisms $X \cong \overline{\bar{X}}$, as 
on the left below.
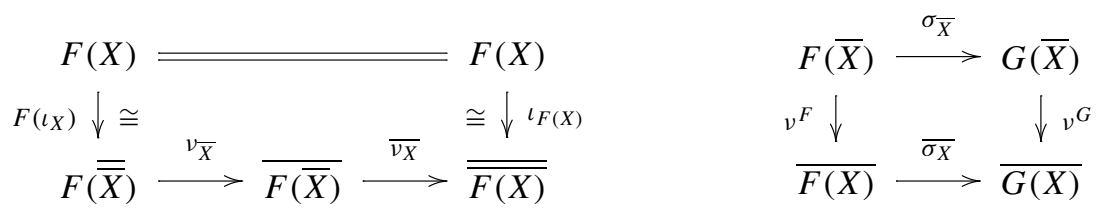

A natural transformation $\sigma: F \Rightarrow G$ between two involutive functors $F, G: \mathbf{C} \rightrightarrows \mathbf{D}$ is called involutive if it commutes with the associated $v$ 's, as on the right above. This yields a 2-category ICat of involutive categories, functors and natural transformations.

This 2-categorical perspective is useful, for instance because it allows us to see immediately what an involutive adjunction or monad is, namely one in which the functors and natural transformations involved are all involutive.

Lemma 2 If $F$ is an involutive functor via $v: F(\bar{X}) \rightarrow \overline{F(X)}$, then this $v$ is automatically an isomorphism.

Proof We construct an inverse for $v$ as composite:

$$
v^{-1} \stackrel{\text { def }}{=}\left(\overline{F(X)} \stackrel{\overline{F(\iota)}}{\longrightarrow} \overline{F(\overline{\bar{X}})} \stackrel{\bar{v}}{\longrightarrow} \overline{\overline{F(\bar{X})}} \stackrel{\iota^{-1}}{\longrightarrow} F(\bar{X})\right)
$$

We explicitly check that this is indeed an inverse to $v$, by using the interaction (2) between $v$ and $\iota$. First we have $v \circ v^{-1}=$ id in:

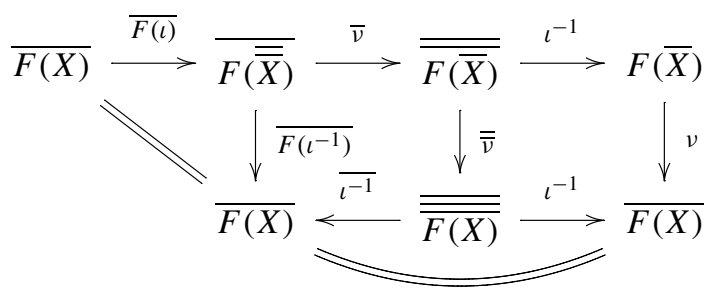

In a similar manner one gets $v^{-1} \circ v=\mathrm{id}$.

\section{Self-conjugates}

Definition 3 For an involutive category $\mathbf{C}$, let $S C(\mathbf{C})$ be the category of selfconjugates in $\mathbf{C}$. Its objects are maps $j: \bar{X} \rightarrow X$ making the triangle below commute. 

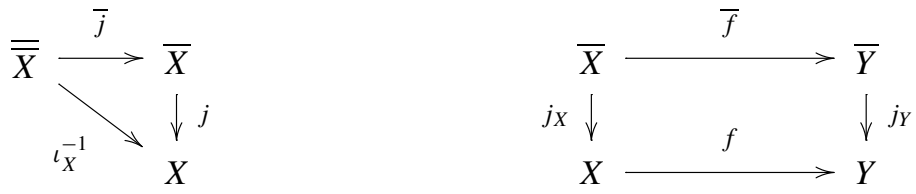

It is not hard to see that such a map $j$ is necessarily an isomorphism, with inverse $\bar{j} \circ \iota_{X}: X \rightarrow \overline{\bar{X}} \rightarrow \bar{X}$.

A morphism $f:\left(X, j_{X}\right) \rightarrow\left(Y, j_{Y}\right)$ in $S C(\mathbf{C})$ is a map $f: X \rightarrow Y$ in $\mathbf{C}$ making the above rectangle commute. There is thus an obvious forgetful functor $\operatorname{SC}(\mathbf{C}) \rightarrow \mathbf{C}$.

What we call a self-conjugate object is called a star object in [7]. By the selfadjointness of Lemma 1 a self-conjugate $\bar{X} \rightarrow X$ may also be described as $X \rightarrow \bar{X}$. Sometimes we call an object $X$ a self-conjugate when the map $\bar{X} \cong X$ involved is obvious from the context. In linear algebra, with $\bar{X}$ given by conjugation (see before Lemma 1), a map of the form $\bar{X} \rightarrow Y$ is called an 'antilinear' or 'conjugate linear' map.

Lemma 3 For an involutive category $\mathbf{C}$, the category $S C(\mathbf{C})$ of self-conjugates is again involutive, via:

$$
(\bar{X} \stackrel{j}{\longrightarrow} X) \stackrel{\text { def }}{=}(\overline{\bar{X}} \stackrel{\bar{j}}{\longrightarrow} \bar{X})
$$

and the forgetful functor $\mathrm{SC}(\mathbf{C}) \rightarrow \mathbf{C}$ is an involutive functor, via the identity natural transformation (as ' $v$ ' in Definition 2).

Proof The map $\iota_{X}: X \stackrel{\cong}{\bar{X}}$ in $\mathbf{C}$ is also a map in $S C(\mathbf{C})$ in:

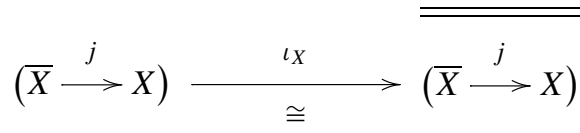

since the following diagram commutes by naturality.

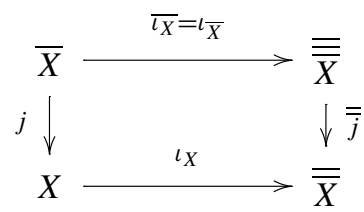

Example 1 Recall that the category PoSets of posets and monotone functions is involutive via the reversed (opposite) order: $\overline{(X, \leq)}=(X, \geq)$. The poset of integers $\mathbb{Z}$ is then self-conjugate, via minus $-: \overline{\mathbb{Z}} \cong \mathbb{Z}$. Also the positive rational and real 
numbers $\mathbb{Q}_{>0}$ and $\mathbb{R}_{>0}$ are self-conjugates in PoSets, via $x \mapsto \frac{1}{x}$. Similarly, for a Boolean algebra $B$, negation $\neg$ yields a self-conjugate $\neg: \bar{B} \cong B$ in the category of Boolean algebras. There are similar self-conjugates via orthosupplements $(-)^{\perp}$ in orthomodular lattices [20] and effect algebras [12].

In Cat a self-conjugate is given by a self-dual category $\mathbf{C}^{\mathrm{op}} \cong \mathbf{C}$.

Recall the conjugation on vector spaces. Suppose $V \in \operatorname{Vect}_{\mathbb{C}}$ has a basis $\left(v_{i}\right)_{i \in I}$. Then we can define a self-conjugate $\bar{V} \cong V$ by $x=\left(\sum_{i} x_{i} v_{i}\right) \longmapsto\left(\sum_{i} \overline{x_{i}} v_{i}\right)$.

Finally, if a category $\mathbf{C}$ is considered with trivial involution $\bar{X}=X$, then $\operatorname{SC}(\mathbf{C})$ contains the self-inverse endomaps $j: X \rightarrow X$, with $j \circ j=\operatorname{id}_{X}$.

We first take a closer look at these trivial involutions.

Lemma 4 Let $\mathbf{C}$ be an ordinary category, considered as involutive with trivial involution $\bar{X}=X$. Assuming binary coproducts + and products $\times$ exist in $\mathbf{C}$, there are left and right adjoints to the forgetful functor:

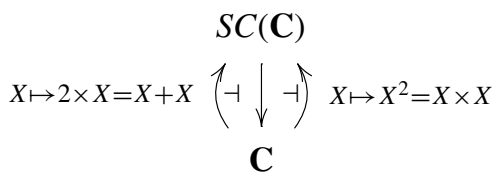

using the swap maps $\left[\kappa_{2}, \kappa_{1}\right]: X+X \stackrel{\cong}{\longrightarrow} X+X$ and $\left\langle\pi_{2}, \pi_{1}\right\rangle: X \times X \stackrel{\cong}{\longrightarrow} X \times X$ as self-conjugates.

Proof Recall that for the trivial involution on $\mathbf{C}$, an object $(Y, j) \in S C(\mathbf{C})$ consists of an isomorphism $j: Y \stackrel{\cong}{\longrightarrow} Y$ with $j^{-1}=j$. For the left adjoint the required bijective correspondence:

$\frac{\left(X+X,\left[\kappa_{2}, \kappa_{1}\right]\right) \stackrel{f}{\longrightarrow}(Y, j)}{X \underset{g}{\longrightarrow} Y} \quad \begin{array}{ll}\text { in } S C(\mathbf{C}) \\ \text { in } \mathbf{C}\end{array}$

exists because the requirement $f \circ\left[\kappa_{2}, \kappa_{1}\right]=j \circ f$ means $f \circ \kappa_{2}=j \circ f \circ \kappa_{1}$. Hence $f$ is determined by $f \circ \kappa_{1}: X \rightarrow Y$. The argument works similarly for the right adjoints, given by products.

Lemma 5 Let $\mathbf{C}$ be an involutive category; $S C(\mathbf{C})$ inherits all limits and colimits that exist in $\mathbf{C}$, and the forgetful functor $\mathrm{SC}(\mathbf{C}) \rightarrow \mathbf{C}$ preserves them.

Proof We give an exemplaric sketch for binary products $\times$. The product of two objects $\left(X, j_{X}\right),\left(Y, j_{Y}\right) \in S C(\mathbf{C})$ is given by:

$$
\overline{X \times Y} \stackrel{\left\langle\overline{\pi_{1}}, \overline{\pi_{2}}\right\rangle}{\cong} \bar{X} \times \bar{Y} \stackrel{j_{X} \times j_{Y}}{\longrightarrow} X \times Y,
$$


where the (canonical) isomorphism on the left exists since $\overline{(-)}$ preserves products, by Lemma 1. It is not hard to see that this is a self-conjugate, forming a product in $\operatorname{SC}(\mathbf{C})$.

For the record we note the following (see $[8,25]$ for background).

Lemma 6 The mapping $\mathbf{C} \mapsto S C(\mathbf{C})$ is a 2-functor ICat $\rightarrow$ ICat, and even a 2comonad.

Proof Essentially this says that we can lift involutive functors and natural transformations as in:

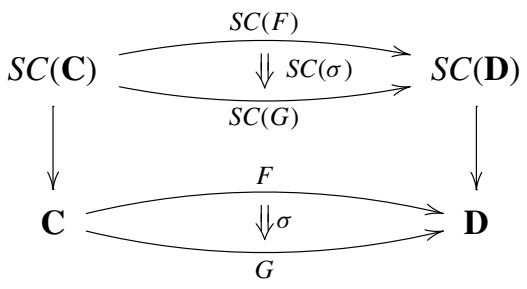

Using Lemma 2 the lifted functor $S C(F)$ is defined as:

$$
(\bar{X} \stackrel{j}{\rightarrow} X) \longmapsto\left(\overline{F(X)} \stackrel{v^{-1}}{\rightarrow} F(\bar{X}) \stackrel{F(j)}{\rightarrow} F(X)\right) .
$$

It is not hard to see that the right-hand-side is a again a self-conjugate. The natural transformation $S C(\sigma)$ on $\bar{X} \rightarrow X$ is simply $\sigma_{X}$.

The counit of $S C$ as 2-comonad is the forgetful functor $S C(\mathbf{C}) \rightarrow \mathbf{C}$, which is natural, see (4). The comultiplication $S C(\mathbf{C}) \rightarrow S C(S C(\mathbf{C}))$ is given by:

$$
(\bar{X} \stackrel{j}{\longrightarrow} X) \longmapsto((\bar{X} \stackrel{j}{\longrightarrow} X) \stackrel{j}{\longrightarrow}(\bar{X} \stackrel{j}{\longrightarrow} X)) .
$$

\section{Involutive Monoidal Categories}

Definition 4 An involutive monoidal category or an involutive symmetric monoidal category, abbreviated as IMC or ISMC, is a category $\mathbf{C}$ which is both involutive and (symmetric) monodial in which involution $\overline{(-)}: \mathbf{C} \rightarrow \mathbf{C}$ is a (symmetric) monoidal functor and the map $\iota$ : id $\Rightarrow \overline{\overline{(-)}}$, satisfying (1), is a monoidal natural transformation.

The fact that involution is a (symmetric) monoidal functor means that there are (natural) maps $\zeta: I \rightarrow \bar{I}$ and $\xi: \bar{X} \otimes \bar{Y} \rightarrow \overline{X \otimes Y}$ commuting with the monoidal isomorphisms $\alpha: X \otimes(Y \otimes Z) \stackrel{\cong}{\longrightarrow}(X \otimes Y) \otimes Z, \lambda: I \otimes X \stackrel{\cong}{\longrightarrow} X, \rho: X \otimes I \rightarrow X$, 
and also with the swap map $\gamma: X \otimes Y \stackrel{\cong}{\longrightarrow} Y \otimes X$ in the symmetric case. That the isomorphism $\iota$ is monoidal means that we have commuting diagrams:
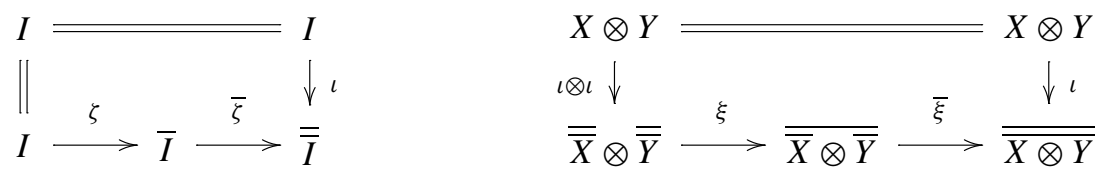

Like in Lemma 2 we get isomorphy for free.

Lemma 7 In an IMC the involution functor $\overline{(-)}$ is automatically strong monoidal: the maps $\zeta: I \rightarrow \bar{I}$ and $\xi: \bar{X} \otimes \bar{Y} \rightarrow \overline{X \otimes Y}$ are necessarily isomorphisms.

Proof All this follows from the requirement $\iota=\bar{\iota}: \bar{X} \rightarrow \overline{\bar{X}}$ in (1) in Definition 1 and the monoidal requirements (6). For instance, the obvious candidate as inverse for $\zeta: I \rightarrow \bar{I}$ is $\iota^{-1} \circ \bar{\zeta}: \bar{I} \rightarrow \overline{\bar{I}} \cong I$. Because $\iota$ is a monoidal natural transformation, we immediately get $\iota^{-1} \circ \bar{\zeta} \circ \zeta=\iota^{-1} \circ \iota=\mathrm{id}$. By post-composing with the isomorphism $\iota=\bar{\iota}: \bar{I} \rightarrow \overline{\bar{I}}$ we get by (6):

$$
\iota \circ \zeta \circ \iota^{-1} \circ \bar{\zeta}=\overline{\bar{\zeta}} \circ \iota \circ \iota^{-1} \circ \bar{\zeta}=\overline{\bar{\zeta} \circ \zeta}=\bar{\iota}=\iota
$$

Similarly, the (candidate) inverse for $\xi: \bar{X} \otimes \bar{Y} \rightarrow \overline{X \otimes Y}$ is:

$$
\overline{X \otimes Y} \stackrel{\overline{\iota \otimes \imath}}{\longrightarrow} \overline{\bar{X}} \otimes \overline{\bar{Y}} \stackrel{\bar{\xi}}{\longrightarrow} \overline{\overline{\bar{X} \otimes \bar{Y}}} \stackrel{\iota^{-1}}{\longrightarrow} \bar{X} \otimes \bar{Y}
$$

In the category Vect $\mathbb{C}_{\mathbb{C}}$ of vector spaces over the complex number the tensor unit $I$ is $\mathbb{C} \in$ Vect $_{\mathbb{C}}$. The above map $\zeta: \mathbb{C} \cong \mathbb{C}$ is simply conjugation of complex numbers.

Remark 1 The notions of 'bar category' in [7] and 'involutive monoidal category' in [13] are similar to the above notion of IMC (or ISMC), but are subtly different: by definition, bar categories have isomorphisms $\overline{X \otimes Y} \cong \bar{Y} \otimes \bar{X}$. The object reversal involved makes sense in a non-symmetric setting; it corresponds to the "reversing" version of involutive monoid described in Definition 6. But in the present context all our examples are symmetric, and many results rely on symmetry, so we often assume it and thus have no difference with [7, 13].

In order to be complete we also have to define the following.

Definition 5 A functor $F: \mathbf{C} \rightarrow \mathbf{D}$ between IMC's is called involutive monoidal if it is both involutive, via $v: F(\bar{X}) \rightarrow \overline{F(X)}$, and monoidal, via $\zeta^{F}: I \rightarrow F(I)$ and $\xi^{F}: F(X) \otimes F(Y) \rightarrow F(X \otimes Y)$, and these natural transformations $v, \zeta^{F}, \xi^{F}$ interact 
appropriately with $\zeta, \xi$ from (6), as in:
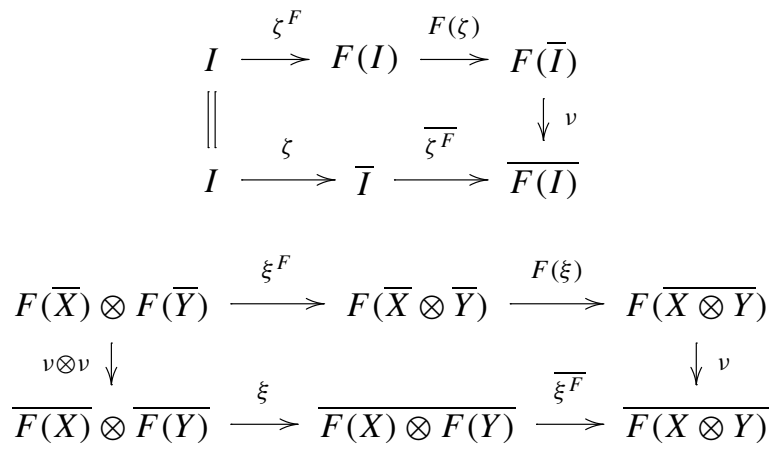

It should then be obvious what an involutive symmetric monoidal functor is.

An involutive monoidal natural transformation $\sigma: F \Rightarrow G$ between two involutive monoidal functors is both involutive and monoidal.

Hence also in this case we have 2-categories IMCat and IMSCat of involutive (symmetric) monoidal categories. The following is the main result of this section.

Proposition 1 A category $S C(\mathbf{C})$ inherits (symmetric) monoidal structure from $\mathbf{C}$. As a result, the forgetful functor $\mathrm{SC}(\mathbf{C}) \rightarrow \mathbf{C}$ is an involutive (symmetric) monoidal functor. In case $\mathbf{C}$ is monoidal closed, then so is $S C(\mathbf{C})$ and $S C(\mathbf{C}) \rightarrow \mathbf{C}$ preserves the exponent $\multimap$.

Proof The tensor unit $I \in \mathbf{C}$ is a self-conjugate via $\zeta^{-1}: \bar{I} \cong I$. If we have selfconjugates $j_{X}: \bar{X} \cong X$ and $j_{Y}: \bar{Y} \cong Y$ we obtain a tensored self-conjugate using Lemma 7:

$$
\overline{X \otimes Y} \underset{\cong}{\stackrel{\xi^{-1}}{\cong}} \bar{X} \otimes \bar{Y} \underset{\cong}{\stackrel{j_{X} \otimes j_{Y}}{\longrightarrow}} X \otimes Y
$$

It is not hard to see that, with this tensor product, the monoidal isomorphisms $\alpha, \lambda, \rho, \gamma$ from $\mathbf{C}$ are also maps in $S C(\mathbf{C})$. Similarly, for the required maps making the involution $\overline{(-)}: S C(\mathbf{C}) \rightarrow S C(\mathbf{C})$ from Lemma 3 into a monoidal functor, we can take the ones from $\mathbf{C}$, in:

$$
\left(\bar{I} \underset{\zeta^{-1}}{\rightarrow} I\right) \stackrel{\zeta}{\longrightarrow} \overline{\left(\bar{I} \underset{\zeta^{-1}}{\longrightarrow} I\right)} \quad \overline{\left(\bar{X} \underset{j_{X}}{\longrightarrow} X\right)} \otimes \overline{\left(\bar{Y} \underset{j_{Y}}{\rightarrow} Y\right)} \stackrel{\xi}{\longrightarrow} \overline{\left(\overline{X \otimes Y} \underset{j_{X} \otimes j_{Y} \circ \xi^{-1}}{\longrightarrow} X \otimes Y\right)} .
$$

The exponent of $\left(X, j_{X}\right),\left(Y, j_{Y}\right) \in S C(\mathbf{C})$ is $X \multimap Y$ with self-conjugate $\overline{X \multimap Y} \rightarrow$ $X \multimap Y$ obtained by abstraction from:

$$
X \otimes \overline{(X \multimap Y)} \stackrel{j_{X}^{-1} \otimes \mathrm{id}}{\longrightarrow} \bar{X} \otimes \overline{(X \multimap Y)} \stackrel{\xi}{\rightarrow} \overline{X \otimes(X \multimap Y)} \stackrel{\overline{e v}}{\rightarrow} \bar{Y} \stackrel{j_{Y}}{\rightarrow} Y .
$$




\section{Involutive Monoids}

By using an involutive category as ambient category, we can define the notion of involutive monoid, in the style of [14-16].

We start with some preliminary observations. Let $M=(M, \cdot, 1)$ be an arbitrary monoid (in Sets), not necessarily commutative. An involution on $M$ is a special endofunction $M \rightarrow M$ which we shall write as superscript negation $x^{-}$, for $x \in M$. It satisfies $x^{--}=x$ and $1^{-}=1$. The interaction of involution and multiplication may happen in two ways: either in a "reversing" manner, as in $(x \cdot y)^{-}=y^{-} \cdot x^{-}$, or in a "non-reversing" manner: $(x \cdot y)^{-}=x^{-} \cdot y^{-}$. Obviously, in a commutative monoid there is no difference between a reversing or non-reversing involution.

As we have argued in the first section via the example of integers in PoSets, a proper formulation of the notion of involutive monoid requires an involutive category, so that the monoid involution can be described as a map $\bar{M} \rightarrow M$.

Definition 6 Let $\mathbf{C}$ be an involutive symmetric monoidal category. An involutive monoid in $\mathbf{C}$ consists of a monoid $I \stackrel{u}{\rightarrow} M \stackrel{m}{\leftarrow} M \otimes M$ in $\mathbf{C}$ together with an involution map $\bar{M} \stackrel{J}{\rightarrow} M$ satisfying:
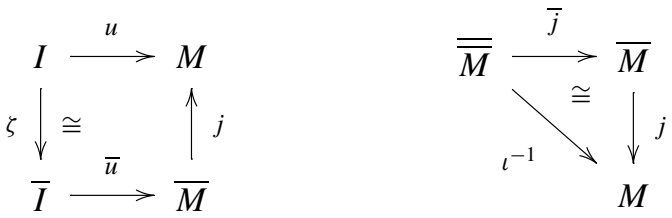

and, one of the following diagrams:
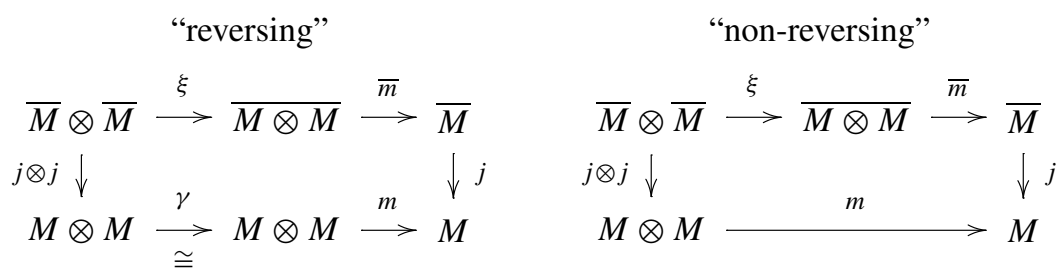

One may call $M$ a simple involutive monoid if C's involution $\overline{(-)}$ is the identity.

A morphism of involutive monoids $M \rightarrow M^{\prime}$ is a morphism of monoids $f: M \rightarrow$ $M^{\prime}$ satisfying $f \circ j=j^{\prime} \circ \bar{f}$. This yields two subcategories $\operatorname{rIMon}(\mathbf{C}) \hookrightarrow \operatorname{Mon}(\mathbf{C})$ and $\operatorname{IMon}(\mathbf{C}) \hookrightarrow \operatorname{Mon}(\mathbf{C})$ of reversing and non-reversing involutive monoids. There is also a commutative version, forming a (full) subcategory. $\operatorname{ICMon}(\mathbf{C}) \hookrightarrow \operatorname{IMon}(\mathbf{C})$.

The involution map $j: \bar{M} \rightarrow M$ of an involutive monoid is of course a selfconjugate-see Definition 3-and thus an isomorphism. In fact, we have the following result.

Lemma 8 Involutive monoids (of the non-reversing kind) are ordinary monoids in the category of self-conjugates: the categories $\operatorname{IMon}(\mathbf{C})$ and $\operatorname{Mon}(\operatorname{SC}(\mathbf{C}))$ are the same. Similarly in the commutative case, $\operatorname{ICMon}(\mathbf{C})=C M o n(\operatorname{SC}(\mathbf{C}))$. 
Proof Since the tensors of $\mathbf{C}$ and $S C(\mathbf{C})$ coincide - see Proposition 1-we only need to check that the above definition precisely says that the unit $u$ and multiplication $m$ of an involutive monoid are maps in $S C(\mathbf{C})$ of the form:

$$
\left(\bar{I}^{-1} \rightarrow I\right) \stackrel{u}{\longrightarrow}(\bar{M} \stackrel{j}{\rightarrow} M) \stackrel{m}{\longleftarrow}^{\longrightarrow}(\bar{M} \stackrel{j}{\rightarrow} M) \otimes\left(\bar{M}^{j} \rightarrow M\right) .
$$

The unit $u$ is a map as indicated on the left if and only if $j \circ \bar{u}=u \circ \zeta^{-1}$. This is precisely the first square in Definition 6. Similarly, $m$ is map on the right if and only if $m \circ(j \otimes j) \circ \xi^{-1}=j \circ \bar{m}$. Again, this is exactly the (non-reversing) requirement in Definition 6.

This lemma suggests a pattern for defining an involutive variant of certain categorical structures, namely by defining this structure in the category of self-conjugates.

Example 2 As we have observed before, the category PoSets of posets and monotone functions is involutive, via order-reversal $\overline{(X, \leq)}=(X, \geq)$. The poset $\mathbb{Z}$ of integers forms an involutive monoid in PoSets, with minus $-: \overline{\mathbb{Z}} \rightarrow \mathbb{Z}$ as involution. Also, the positive rationals $\mathbb{Q}_{>0}$ or reals $\mathbb{R}_{>0}$ with multiplication $\cdot$, unit 1 , and inverse $(-)^{-1}$ form involutive monoids in PoSets.

In the category Cat of categories, with finite products as monoidal structure, a monoid is a strictly monoidal category. If such a category $\mathbf{C}$ has a dagger $\dagger: \mathbf{C}^{\mathrm{op}} \rightarrow \mathbf{C}$ that commutes with these tensors (in the sense that $(f \otimes g)^{\dagger}=f^{\dagger} \otimes g^{\dagger}$, see e.g. [2]) then $\mathbf{C}$ is an involutive monoid in Cat.

Inside such a dagger symmetric (not necessarily strict) monoidal category $\mathbf{C}$ with dagger $(-)^{\dagger}: \mathbf{C o p} \rightarrow \mathbf{C}$ the homset of scalars $I \rightarrow I$ is a commutative involutive monoid, with involution $s^{-}=s^{\dagger}$.

The tensor unit $I \in \mathbf{C}$ in an arbitrary involutive category $\mathbf{C}$ is a commutative involutive monoid object, with involution $\zeta^{-1}: \bar{I} \rightarrow I$.

We briefly describe free involutive monoids in the category Sets (with trivial involution), both of the reversing and non-reversing kind. We recall that the set $V^{\star}$ of finite lists $\left\langle v_{1}, \ldots, v_{n}\right\rangle$ of elements $v_{i} \in V$, is the free monoid on a set $V$, with empty list \langle\rangle as unit and concatenation of lists as composition. We shall write 2 for the twoelement set $2=\{-,+\}$ of signs with negation (or involution) $-: 2 \rightarrow 2$ given by $--=+$ and $-+=-$.

Proposition 2 The free non-reversing involutive monoid on $V \in$ Sets is the set $(2 \times$ $V)^{\star}$ of "signed" lists, with involution:

$$
\left\langle\left(b_{1}, v_{1}\right), \ldots,\left(b_{n}, v_{n}\right)\right\rangle^{-}=\left\langle\left(-b_{1}, v_{1}\right), \ldots,\left(-b_{n}, v_{n}\right)\right\rangle,
$$

where $b_{i} \in 2$ and $v_{i} \in V$. The free reversing involutive monoid also has $(2 \times V)^{\star}$ as carrier, but now with involution involving list reversal:

$$
\left\langle\left(b_{1}, v_{1}\right), \ldots,\left(b_{n}, v_{n}\right)\right\rangle^{-}=\left\langle\left(-b_{n}, v_{n}\right), \ldots,\left(-b_{1}, v_{1}\right)\right\rangle .
$$

In both cases we use $\eta(v)=\langle(+, v)\rangle$ as insertion $\eta: V \rightarrow(2 \times V)^{\star}$. 
Proof Given an involutive monoid $M=\left(M, 1, \cdot,(-)^{-}\right)$in Sets, a map $f: V \rightarrow M$ can be extended in a unique way to a map of non-reversing involutive monoids $\widehat{f}:(2 \times V)^{\star} \rightarrow M$, via

$$
\widehat{f}\left(\left\langle\left(b_{1}, v_{1}\right), \ldots,\left(b_{n}, v_{n}\right)\right\rangle\right)=f\left(v_{1}\right)^{b_{1}} \ldots . f\left(v_{n}\right)^{b_{n}},
$$

where for $x \in M$ we write $x^{+}=x$ and $x^{-}$for the result of applying $M$ 's involution $(-)^{-}$to $x$. Clearly, $\widehat{f}$ preserves the unit and composition, and satisfies $\widehat{f} \circ \eta=f$. In order to make the differences between 'reversing' and 'non-reversing' explicit, we write out the details. In the non-reversing case $\widehat{f}$ preserves the involution:

$$
\begin{aligned}
\widehat{f}\left(\left\langle\left(b_{1}, v_{1}\right), \ldots,\left(b_{n}, v_{n}\right)\right\rangle^{-}\right) & \left.=\widehat{f}\left\langle\left(-b_{1}, v_{1}\right), \ldots,\left(-b_{n}, v_{n}\right)\right\rangle\right) \\
& =f\left(v_{1}\right)^{-b_{1}} \cdots f\left(v_{n}\right)^{-b_{n}} \\
& =\left(f\left(v_{1}\right)^{b_{1}}\right)^{-} \cdots\left(f\left(v_{n}\right)^{b_{n}}\right)^{-} \\
& =\left(f\left(v_{1}\right)^{b_{1}} \cdots f\left(v_{n}\right)^{b_{n}}\right)^{-} \\
& =\left(\widehat{f}\left(\left\langle\left(b_{1}, v_{1}\right), \ldots,\left(b_{n}, v_{n}\right)\right\rangle\right)\right)^{-} .
\end{aligned}
$$

Similarly in the reversing case involution is preserved, because:

$$
\begin{aligned}
\widehat{f}\left(\left\langle\left(b_{1}, v_{1}\right), \ldots,\left(b_{n}, v_{n}\right)\right\rangle^{-}\right) & \left.=\widehat{f}\left\langle\left(-b_{n}, v_{n}\right), \ldots,\left(-b_{1}, v_{1}\right)\right\rangle\right) \\
& =f\left(v_{n}\right)^{-b_{n}} \cdots f\left(v_{1}\right)^{-b_{1}} \\
& =\left(f\left(v_{n}\right)^{b_{n}}\right)^{-} \cdots\left(f\left(v_{1}\right)^{b_{1}}\right)^{-} \\
& =\left(f\left(v_{1}\right)^{b_{1}} \cdots f\left(v_{n}\right)^{b_{n}}\right)^{-} \\
& =\left(\widehat{f}\left(\left\langle\left(b_{1}, v_{1}\right), \ldots,\left(b_{n}, v_{n}\right)\right\rangle\right)\right)^{-} .
\end{aligned}
$$

\section{Involutions and Algebras}

This section briefly discusses involutions on monads and will focus on algebras of such monads. Familiarity with the basics of the theory of monads is assumed, see e.g. $[6,23,24]$. The following definition makes explicit what it means to be a monad in the 2-category of involutive categories.

Definition 7 Let $T=(T, \eta, \mu)$ be a monad on an involutive category $\mathbf{C}$. We shall call $T$ an involutive monad if $T: \mathbf{C} \rightarrow \mathbf{C}$ is an involutive functor, say via $v_{X}: T(\bar{X}) \rightarrow$ $\overline{T(X)}$, and the unit $\eta$ and multiplication $\mu$ are involutive natural transformations. As a result, $v$ forms a distributive law of the monad $T$ over $\mathbf{C}$ 's involution $\overline{(-)}$. This 
amounts to:
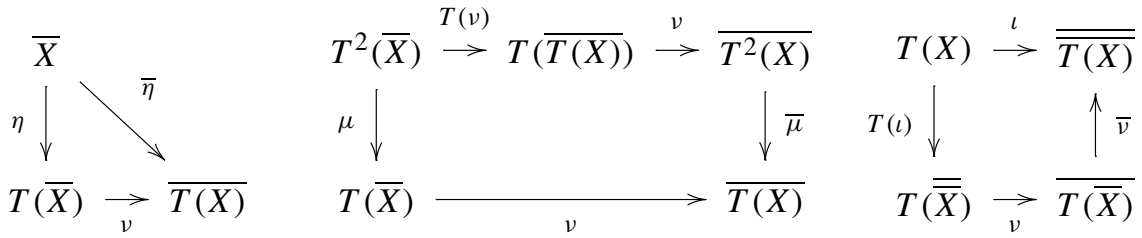

This monad is called involutive (symmetric) monoidal if $T$ and $\eta, \mu$ are involutive (symmetric) monoidal.

With respect to the identity involution on a (symmetric monoidal) category $\mathbf{C}$, any monad is involutive via the identity distributive law. But the identity involution on a category may still give rise to meaningful involutive monads, as the semiring example below shows.

Example 3 (i) Let $M=(M, m, u, j)$ be an involutive (non-reversing) monoid in an involutive category $\mathbf{C}$. As is well-known the functor $M \otimes(-): \mathbf{C} \rightarrow \mathbf{C}$ is a monad; its unit and multiplication are:

$$
X \underset{\lambda^{-1}}{\stackrel{\longrightarrow}{\longrightarrow}} I \otimes X \stackrel{u \otimes \mathrm{id}}{\longrightarrow} M \otimes X \quad M \otimes(M \otimes X) \underset{\cong}{\stackrel{\alpha}{\longrightarrow}}(M \otimes M) \otimes X \stackrel{m \otimes \mathrm{id}}{\longrightarrow} M \otimes X .
$$

Unsurprisingly, $M$ 's involution $j$ makes this an involutive monad via:

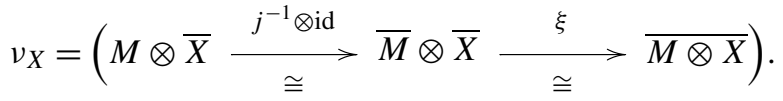

(ii) Let $S$ be an involutive commutative semiring, i.e. a commutative semiring with an endomap $(-)^{-}: S \rightarrow S$ that is a semiring homomorphism with $s^{--}=s$. An obvious example is the set $\mathbb{C}$ of complex numbers with conjugation $\overline{a+i b}=a-i b$. Similarly, the Gaussian rational numbers (with $a, b \in \mathbb{Q}$ in $a+i b$ ) form an involutive semiring, albeit not a complete one.

Consider the multiset monad $\mathcal{M}_{S}$ : Sets $\rightarrow$ Sets associated with $S$, where we use Sets as trivial involutive category, with the identity as involution. This monad is defined on a set $X$ as:

$$
\mathcal{M}_{S}(X)=\{\varphi: X \rightarrow S \mid \operatorname{supp}(\varphi) \text { is finite }\} .
$$

Such a multiset $\varphi \in \mathcal{M}_{S}(X)$ may be written as formal sum $s_{1} x_{1}+\cdots+s_{k} x_{k}$ where $\operatorname{supp}(\varphi)=\left\{x_{1}, \ldots, x_{k}\right\}$ and $s_{i}=\varphi\left(x_{i}\right) \in S$ describes the "multiplicity" of the element $x_{i} \in X$. For more information, see e.g. [11]. The category of algebras of this monad is the category $\operatorname{Mod}_{S}$ of modules over $S$.

This monad is monoidal / commutative, because $S$ is commutative. It is involutive, with involution $v: \mathcal{M}_{S}(X) \rightarrow \mathcal{M}_{S}(X)$ given by $v\left(\sum_{i} s_{i} x_{i}\right)=\sum_{i} s_{i}^{-} x_{i}$. 
For an involutive monad $T$ on an involutive category $\mathbf{C}$ we can consider two liftings, namely of the monad $T$ to self-conjugates $S C(\mathbf{C})$ following Lemma 6, or of $\mathbf{C}$ 's involution $\overline{(-)}$ to algebras $A \lg (T)$, as in the following two diagrams.
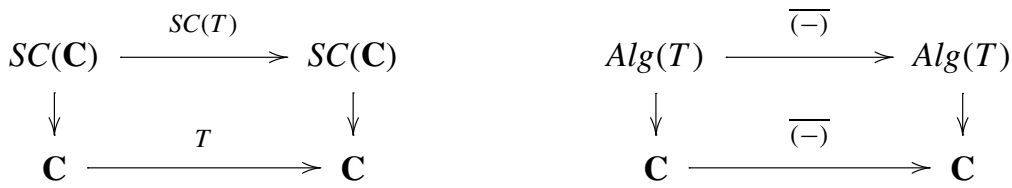

The lifting on the left yields a new monad $S C(T)$ because lifting in Lemma 6 is 2-functorial. The lifting on the right arises because an involutive monad involves a distributive law commuting with unit and multiplication. Explicitly, it is given by:

$$
(T(X) \stackrel{a}{\longrightarrow} X) \stackrel{\text { def }}{=}\left(T(\bar{X}) \stackrel{v_{X}}{\longrightarrow} \overline{T(X)} \stackrel{\bar{a}}{\longrightarrow} \bar{X}\right) .
$$

Proposition 3 Suppose $T$ is an involutive monad on an involutive category $\mathbf{C}$. The category $\operatorname{Alg}(T)$ is then also involutive via (8), and:

1. $A \lg (S C(T))=S C(A \lg (T))$, for which one may write $\operatorname{IAlg}(T)$;

2. the canonical adjunction $\operatorname{Alg}(T) \leftrightarrows \mathbf{C}$ is an involutive one.

Proof Definition (8) yields a new algebra because $v$ is a distributive law. The involution map $\operatorname{id}_{A l g(T)} \Rightarrow \overline{\overline{(-)}}$ on algebras is given by $\mathbf{C}$ 's involution map $\iota$, in:

$$
(T(X) \stackrel{a}{\longrightarrow} X) \stackrel{\iota}{\cong}(T(X) \stackrel{a}{\longrightarrow} X)
$$

It is not hard to see that $\iota$ is a map of algebras. The involution functor $\operatorname{Alg}(T) \rightarrow$ $\operatorname{Alg}(T)$ on a morphism $f$ of algebras is just $\bar{f}$.

For point 1 notice that on the one hand an $S C(T)$-algebra is a map

$$
\operatorname{SC}(T)\left(\bar{X} \stackrel{j_{X}}{\rightarrow} X\right) \stackrel{a}{\longrightarrow}\left(\bar{X} \stackrel{j_{X}}{\rightarrow} X\right)
$$

which is a $T$-algebra $a: T(X) \rightarrow X$ that is a map of self-conjugates, using (5) on the left in:

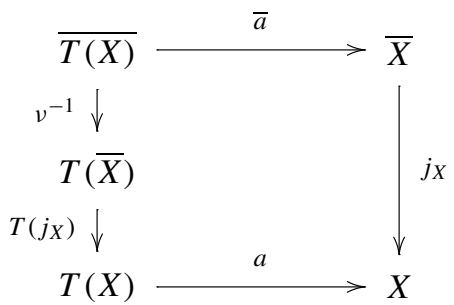


On the other hand a self-conjugate in $\operatorname{Alg}(T)$ consists of an algebra $a$ with a map of the form:

$$
(T(X) \stackrel{a}{\longrightarrow} X) \stackrel{j_{X}}{\longrightarrow}(T(X) \stackrel{a}{\longrightarrow} X)
$$

which means that $j_{X}$ is a map of algebras:

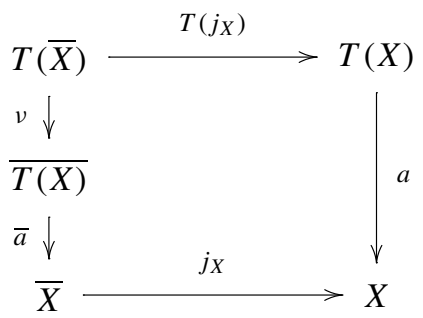

This is evidently the same as the previous rectangle.

As to the second point, the forgetful functor $\operatorname{Alg}(T) \rightarrow \mathbf{C}$ clearly commutes with involution. The free functor $F: \mathbf{C} \rightarrow A \lg (T)$, mapping $X \in \mathbf{C}$ to the algebra $\mu: T^{2}(X) \rightarrow T(X)$, is involutive via the map $F(\bar{X}) \rightarrow \overline{F(X)}$ that is simply $v$ itself by the (second) diagram in Definition 7 , in:

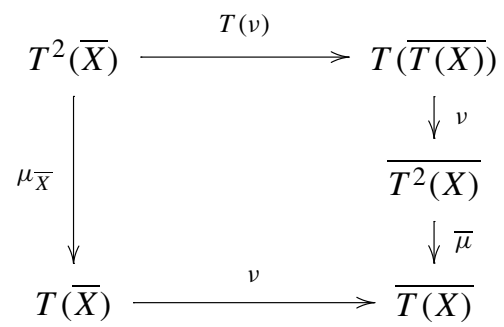

In a next step we would like to show that these categories of algebras of an involutive monoidal monad are also involutive monoidal categories. The monoidal structure is given by the standard construction of Anders Kock [21, 22]. Tensors of algebras exist in case certain colimits exist. This is always the case with monads on sets, due to a result of Linton's, see [6, Sect. 9.3, Prop. 4].

This tensor product $a \bigotimes b=(T X \stackrel{a}{\rightarrow} X) \otimes(T Y \stackrel{b}{\rightarrow} Y)$ of algebras is such that algebra morphisms $a \bigotimes b \rightarrow c$ correspond to bimorphisms [21,22]. The latter can be defined abstractly. This tensor $a \bigotimes b$ arises as coequaliser in the category $\operatorname{Alg}(T)$, of the form:

$$
\left(\begin{array}{c}
T^{2}(T X \otimes T Y) \\
\downarrow_{\mu} \\
T(T X \otimes T Y)
\end{array}\right) \underset{\mu \circ T(\xi)}{\longrightarrow}\left(\begin{array}{c}
T^{2}(X \otimes Y) \\
\downarrow \mu \\
T(X \otimes Y)
\end{array}\right) \longrightarrow\left(\begin{array}{c}
T(X \otimes Y) \\
\downarrow a \otimes b \\
X \otimes Y
\end{array}\right) .
$$

We only give a sketch of the following result. 
Theorem 1 Suppose $T$ is an involutive monoidal monad on an involutive monoidal category $\mathbf{C}$; assume the category Alg $(T)$ of algebras has enough coequalisers to make it monoidal. The category $\operatorname{Alg}(T)$ is then also involutive monoidal, and the canonical adjunction $\operatorname{Alg}(T) \leftrightarrows \mathbf{C}$ is an involutive monoidal one. This result extends to symmetric monoidal structure, and also to closure (with exponents $\multimap$ ).

Proof For algebras $T(X) \stackrel{a}{\rightarrow} X$ and $T(Y) \stackrel{b}{\rightarrow} Y$ we need to obtain a map of algebras $\xi^{A l g(T)}: \bar{a} \otimes \bar{b} \rightarrow \overline{a \otimes b}$ using the universal property described above. The map $\bar{\otimes} \circ$ $\xi^{\mathbf{C}}: \bar{X} \otimes \bar{Y} \rightarrow \overline{X \otimes Y} \rightarrow \overline{X \otimes Y}$ is bilinear map, where $\otimes=t \circ \eta: X \otimes Y \rightarrow X \otimes Y$ is the universal bilinear map. Hence we obtain $\xi^{\operatorname{Alg}(T)}$ with $\xi^{\operatorname{Alg}(T)} \circ \otimes=\bar{\otimes} \circ \xi^{\mathbf{C}}$. The free algebra $F(I)$ is unit for the tensor $\bigotimes$ on $\operatorname{Alg}(T)$ and comes with a map of algebras $\zeta^{A \lg (T)}=v \circ T\left(\zeta^{\mathbf{C}}\right): F(I) \rightarrow \overline{F(I)}$.

The construction (8) gives for an involutive commutative semiring $S$ an involution on the category $\operatorname{Mod}_{S}$ of $S$-modules, which maps a module $X$ to its conjugate space $\bar{X}$, with the same vectors but with scalar multiplication in $\bar{X}$ given by: $s \cdot \bar{X} x=s^{-} \cdot{ }_{X} x$.

Conjugate modules often occur in the context of Hilbert spaces. The category Hilb is indeed an involutive category, via this conjugation. Hence one can consider for instance involutive monoids in Hilb. They are sometimes called (unital) $H^{*}$-algebras.

This section concentrated on algebras of involutive monads. Kleisli categories are also of interest. For an involutive semiring $S$ one can also consider the category Cat Bif $\mathrm{MRel}_{S}$ of $S$-valued "bifinite multirelations". Objects are sets, and morphisms $X \rightarrow Y$ are maps $X \times Y \rightarrow S$ such that the two resulting maps $X \rightarrow S^{Y}$ and $Y \rightarrow S^{X}$ factor as Kleisli maps $X \rightarrow \mathcal{M}_{S}(Y)$ and $Y \rightarrow \mathcal{M}_{S}(X)$. This yields a dagger category with (dagger) tensors and biproducts, whose set of scalars is $S$ itself. See [19], and also [9, 18], for more details.

\section{Inner Product Spaces and the GNS-Construction}

In this final section we consider inner product spaces $V$, with an inner product operation $\langle-\mid-\rangle: \bar{V} \otimes V \rightarrow X$. The input type $\bar{V} \otimes V$ of this operation incorporates anti-linearity in the first argument and linearity in the second one. We wish to apply the theory developed so far to obtain a part of the (unital version of the) GelfandNaimark-Segal (GNS) construction [3], giving a bijective correspondence between states on $C^{*}$-algebras and certain sesquilinear maps. Abstractly, this takes the form of an isomorphism between categories in Theorem 2 below. More concretely, for an involutive monoid $A$ in the category $\operatorname{Mod}_{S}$ of modules, a state $f: A \rightarrow S$ gives rise to an inner product $\langle-\mid-\rangle: \bar{A} \otimes A \rightarrow S$ by $\langle a \mid b\rangle=f\left(a^{-} \cdot b\right)$, where $\cdot$ is the multiplication of the monoid $A$. As mentioned, using the involution in the input type $\bar{A} \otimes A$ of the inner product gives a neat way of handling conjugation in the condition $\langle s \cdot a \mid b\rangle=s^{-} \cdot\langle a \mid b\rangle$, where this last $\cdot$ is the (scalar) multiplication of the semiring $S$ (which is the tensor unit in $\operatorname{Mod}_{S}$ ).

We start by definining appropriate categories of inner product spaces. 
Definition 8 Let $\mathbf{C}$ be an involutive symmetric monoidal category (ISMC). We write $I P(\mathbf{C})$ for the category of inner product spaces in $\mathbf{C}$. Objects are given by a selfconjugate $\left(X, j_{X}\right)$ of "inner product values" together with a "space" object $V \in \mathbf{C}$ map of self-conjugates:

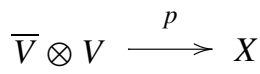

where $\bar{V} \otimes V$ carries the "twist" conjugate $t$ defined as:

$$
t \stackrel{\text { def }}{=}\left(\overline{\bar{V} \otimes V} \stackrel{\overline{\mathrm{id} \otimes \iota V}}{\longrightarrow} \overline{\bar{V} \otimes \overline{\bar{V}}} \stackrel{\bar{\xi}}{\longrightarrow} \overline{\bar{V} \otimes \overline{\bar{V}}} \stackrel{\iota^{-1}}{\longrightarrow} V \otimes \bar{V} \stackrel{\gamma}{\longrightarrow} \bar{V} \otimes V\right) .
$$

A morphism from $\bar{V} \otimes V \stackrel{p}{\rightarrow} X$ to $\bar{W} \otimes W \stackrel{q}{\rightarrow} Y$ in $I P(\mathbf{C})$ is given by a pair of maps: $f:\left(X, j_{X}\right) \rightarrow\left(Y, j_{Y}\right)$ in $S C(\mathbf{C})$ and $g: V \rightarrow W$ in $\mathbf{C}$ making the following diagram commute.

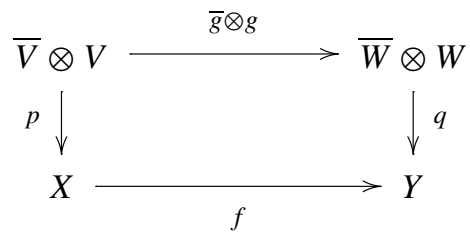

The map $\bar{g} \otimes g$ on top is a morphism of the twist conjugates; hence this is a commuting diagram in $S C(\mathbf{C})$.

There is an obvious forgetful functor $I P(\mathbf{C}) \rightarrow S C(\mathbf{C})$, sending an inner product space $\bar{V} \otimes V \rightarrow X$ to its object of values $X$.

This forgetful functor is an "opfibration" (see [17]) in an obvious sense: via a map $f: X \rightarrow Y$ in $S C(\mathbf{C})$ one can transform inner products on $X$ to inner products on $Y$ via post-composition:

$$
(\bar{V} \otimes V \stackrel{p}{\longrightarrow} X) \longmapsto f_{*}(p) \stackrel{\text { def }}{=}(\bar{V} \otimes V \stackrel{f \circ p}{\longrightarrow} Y) .
$$

There are alternatives to the choice of morphism between inner product spaces used in Definition 8. Especially, instead of a single map $g: V \rightarrow W$ between the spaces involved one can use a pair of maps $W \rightarrow V$ and $V \rightarrow W$ in opposite directions, satisfying an adjointness requirement. However, the above definition works best in the current setting.

We shall use inner product spaces where the space object is a (reversing) involutive monoid and the inner product interacts appropriately with this monoid structure. This can be expressed informally as:

$$
\langle a \cdot b \mid c\rangle=\left\langle a \mid b^{-} \cdot c\right\rangle .
$$

This property is an explicit condition (11) below. 
Definition 9 For an involutive symmetric monoidal category $\mathbf{C}$ the subcategory $M I P(\mathbf{C}) \hookrightarrow I P(\mathbf{C})$ of "monoid inner product spaces" has objects $p: \bar{M} \otimes M \rightarrow X$, where $M=(M, u, m, j)$ is a reversing involutive monoid and $p$ is an inner product space_-in particular, a map of self-conjugates — that additionally makes the following diagram commute.

$$
\begin{gathered}
(\bar{M} \otimes \bar{M}) \otimes M \stackrel{\xi \otimes \mathrm{id}}{\longrightarrow} \overline{(M \otimes M)} \otimes M \stackrel{\bar{m} \otimes \mathrm{id}}{\longrightarrow} \bar{M} \otimes M \stackrel{p}{\longrightarrow} X \\
\gamma \otimes \mathrm{id} \downarrow \cong \\
(\bar{M} \otimes \bar{M}) \otimes M \stackrel{\uparrow p}{\cong} \overline{\alpha^{-1}}
\end{gathered}
$$

A morphism from $\bar{M} \otimes M \stackrel{p}{\rightarrow} X$ to $\bar{N} \otimes N \stackrel{q}{\rightarrow} Y$ in $M I P(\mathbf{C})$ is a pair of maps $f: X \rightarrow$ $Y, g: M \rightarrow N$ in $I P(\mathbf{C})$ where $g$ is also a map of involutive monoids. Again there is a forgetful functor $\operatorname{MIP}(\mathbf{C}) \rightarrow S C(\mathbf{C})$ that is an opfibration like in (10).

The next result relates (reversing) involutive monoids and these monoid inner product spaces.

Proposition 4 There is a functor $\mathcal{F}: \operatorname{rIMon}(\mathbf{C}) \rightarrow M I P(\mathbf{C})$, given by

$$
M \longmapsto \mathcal{F}(M) \stackrel{\text { def }}{=}(\bar{M} \otimes M \stackrel{j \otimes \mathrm{id}}{\longrightarrow} M \otimes M \stackrel{m}{\longrightarrow} M)
$$

in a commuting triangle

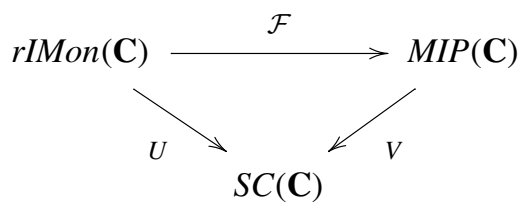

where $U, V$ are forgetful functors.

This functor $\mathcal{F}$ has a right adjoint $\mathcal{G}: \operatorname{MIP}(\mathbf{C}) \rightarrow$ rIMon $(\mathbf{C})$ simply given by:

$$
(\bar{N} \otimes N \stackrel{q}{\longrightarrow} Y) \longmapsto N
$$

Proof We first check that $\mathcal{F}(M)=(m \circ(j \otimes$ id $): \bar{M} \otimes M \rightarrow M)$ satisfies property (11) from Definition 9 . This is easy:

$$
\begin{aligned}
m & \circ(j \otimes \mathrm{id}) \circ(\mathrm{id} \otimes m) \circ(\mathrm{id} \otimes(j \otimes \mathrm{id})) \circ \alpha^{-1} \circ(\gamma \otimes \mathrm{id}) \\
& =m \circ(\mathrm{id} \otimes m) \circ(j \otimes(j \otimes \mathrm{id})) \circ \alpha^{-1} \circ(\gamma \otimes \mathrm{id}) \\
& =m \circ(\mathrm{id} \otimes m) \circ \alpha^{-1} \circ((j \otimes j) \otimes \mathrm{id}) \circ(\gamma \otimes \mathrm{id})
\end{aligned}
$$




$$
\begin{aligned}
& =m \circ(m \otimes \mathrm{id}) \circ(\gamma \otimes \mathrm{id}) \circ((j \otimes j) \otimes \mathrm{id}) \\
& =m \circ(j \otimes \mathrm{id}) \circ(\bar{m} \otimes \mathrm{id}) \circ(\xi \otimes \mathrm{id}), \quad \text { since } M \text { is reversing. }
\end{aligned}
$$

The mapping $M \mapsto \mathcal{F}(M)$ is obviously functorial and commutes with the forgetful functors in the above triangle.

The adjunction $\mathcal{F} \dashv \mathcal{G}$ exists because there is a bijective correspondence:

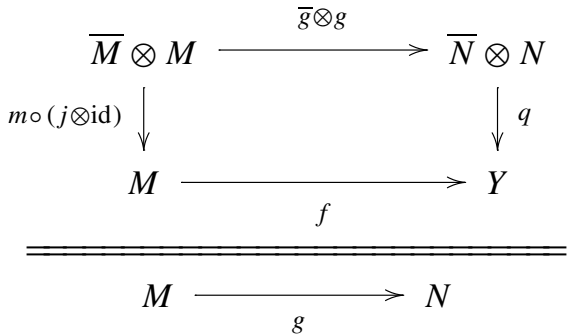

In this situation the map $f$ is completely determined. By precomposing the rectangle above with $((\bar{u} \circ \zeta) \otimes$ id $) \circ \lambda^{-1}: M \rightarrow \bar{M} \otimes M$ one obtains:

$$
\begin{aligned}
f=f \circ m \circ(u \otimes \mathrm{id}) \circ \lambda^{-1} & =f \circ m \circ(j \otimes \mathrm{id}) \circ((\bar{u} \circ \zeta) \otimes \mathrm{id}) \circ \lambda^{-1} \\
& =q \circ(\bar{g} \otimes g) \circ((\bar{u} \circ \zeta) \otimes \mathrm{id}) \circ \lambda^{-1} \\
& =q \circ((\bar{v} \circ \zeta) \otimes g) \circ \lambda^{-1} \\
& =q \circ((\bar{v} \circ \zeta) \otimes \mathrm{id}) \circ \lambda^{-1} \circ g,
\end{aligned}
$$

where $v: I \rightarrow N$ is neutral element of the monoid $N$. It is not hard to check, using property (11), that by defining $f$ in this manner one obtains a morphism $\mathcal{F}(M) \rightarrow q$ in $\operatorname{MIP}(\mathbf{C})$ from $g: M \rightarrow N=\mathcal{G}(q)$ in $\operatorname{rIMon}(\mathbf{C})$.

Theorem 2 Consider the comma category $(U \downarrow S C(\mathbf{C}))$ of the forgetful functor $U: \operatorname{rIMon}(\mathbf{C}) \rightarrow S C(\mathbf{C})$ in the triangle in Proposition 4. It gives rise to an isomorphism of categories in the triangle:

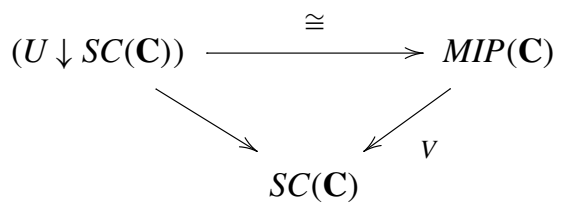

Concretely, for a self-conjugate $X$ and a (reversing) involutive monoid $M$ this gives a (functorial) bijective correspondence between "states" and "inner products":

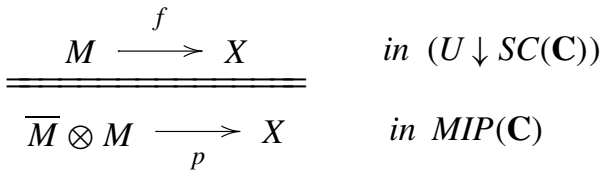


Proof First, there is a functor $\mathcal{H}:(U \downarrow S C(\mathbf{C})) \rightarrow M I P(\mathbf{C})$. For $M \in \operatorname{rIMon}(\mathbf{C})$ and $X \in S C(\mathbf{C})$ it is given by:

$$
(M \stackrel{f}{\longrightarrow} X) \longmapsto f_{*}(\mathcal{F}(M))=(\bar{M} \otimes M \stackrel{j \otimes \text { id }}{\longrightarrow} M \otimes M \stackrel{m}{\longrightarrow} M \stackrel{f}{\longrightarrow} X) .
$$

Here we use the left adjoint $\mathcal{F}$ from Proposition 4 and the reindexing functor $f_{*}$ from (10). This mapping $\mathcal{H}$ is functorial, since if we have $g: M \rightarrow M^{\prime}$ in $\operatorname{rIMon}(\mathbf{C})$ and $h: X \rightarrow X^{\prime}$ in $S C(\mathbf{C})$ in a commuting diagram on the left below, forming a morphism $f \rightarrow f^{\prime}$ in the comma category $(U \downarrow S C(\mathbf{C}))$, then we obtain a map $\mathcal{H}(f) \rightarrow \mathcal{H}\left(f^{\prime}\right)$ in $\operatorname{MIP}(\mathbf{C})$ on the right.
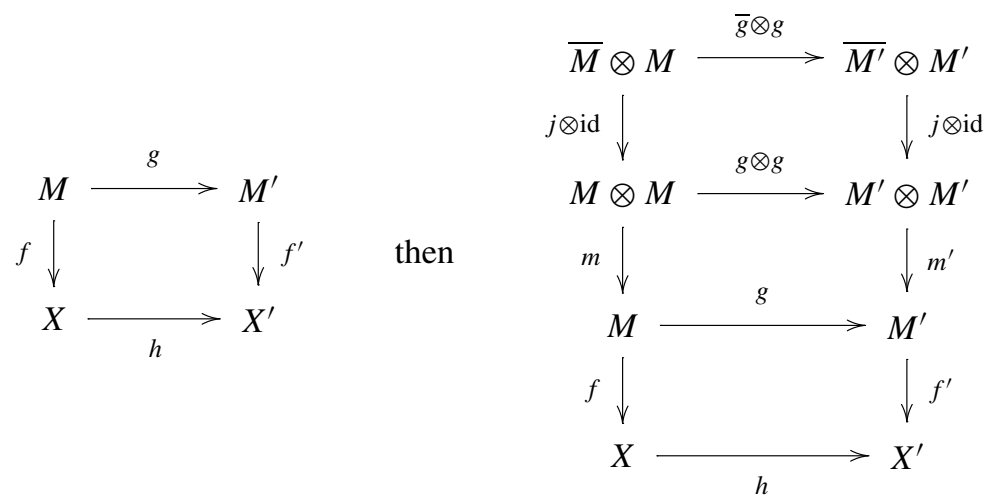

In the reverse direction there is a functor $\mathcal{K}: M I P(\mathbf{C}) \rightarrow(U \downarrow S C(\mathbf{C}))$ by:

$$
(\bar{N} \otimes N \stackrel{q}{\rightarrow} Y) \mapsto \mathcal{K}(q) \stackrel{\text { def }}{=}\left(N \underset{\cong}{\stackrel{\lambda^{-1}}{\longrightarrow}} I \otimes N \stackrel{\zeta \otimes \text { id }}{\rightarrow} \bar{I} \otimes N \stackrel{\bar{v} \otimes \text { id }}{\rightarrow} \bar{N} \otimes N \stackrel{q}{\rightarrow} Y\right) .
$$

Again this is functorial.

We check that $\mathcal{H}$ and $\mathcal{K}$ are each others inverses. We get $\mathcal{K}(\mathcal{H}(f))=f$ in:

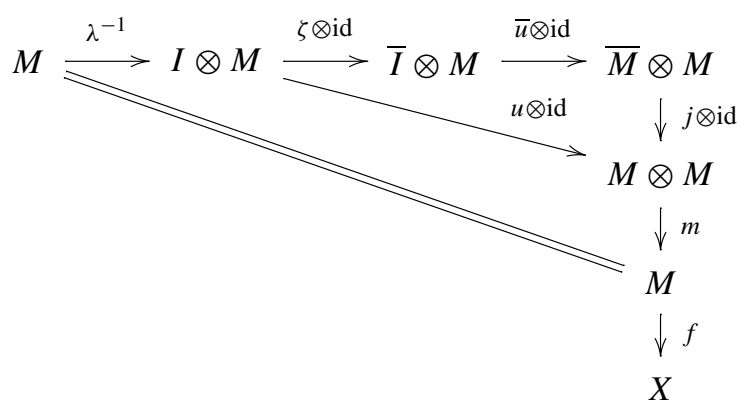


Next we have $\mathcal{H}(\mathcal{K}(q))=q$ because $\mathcal{K}(q)$ is the counit $\mathcal{F}(\mathcal{G}(q)) \rightarrow q$ of the adjunction $\mathcal{F} \dashv \mathcal{G}$ from Proposition 4 in a situation:

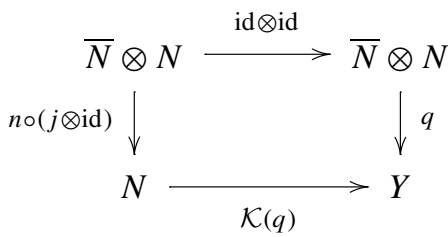

As said, this result only captures part of the GNS construction [3]; it ignores the analytic aspects. The whole construction additionally involves suitable quotients, in order to identify points $a, b$ with $\langle a \mid b\rangle=0$, and completions, in order to get a complete metric space, and thus a Hilbert space.

Acknowledgements Thanks to Chris Heunen, Jorik Mandemaker and the two referees for useful feedback.

Open Access This article is distributed under the terms of the Creative Commons Attribution Noncommercial License which permits any noncommercial use, distribution, and reproduction in any medium, provided the original author(s) and source are credited.

\section{References}

1. Abramsky, S., Blute, R., Panangaden, P.: Nuclear and trace ideals in tensored $*$-categories. J. Pure Appl. Algebra 143, 3-47 (2000)

2. Abramsky, S., Coecke, B.: A categorical semantics of quantum protocols. In: Engesser, K., Gabbai, D.M., Lehmann, D. (eds.) Handbook of Quantum Logic and Quantum Structures, pp. 261-323. North Holland/Elsevier/Computer Science, Amsterdam (2009)

3. Arveson, W.: A Short Course on Spectral Theory. Springer, Berlin (2002)

4. Awodey, S.: Category Theory. Oxford Logic Guides. Oxford Univ. Press, London (2006)

5. Baez, J.C., Dolan, J.: Higher dimensional algebra III: $n$-categories and the algebra of opetopes. Adv. Math. 135, 145-206 (1998)

6. Barr, M., Wells, Ch.: Toposes, Triples and Theories. Springer, Berlin (1985). Revised and corrected version available from URL: www.cwru.edu/artsci/math/wells/pub/ttt.html

7. Beggs, E.J., Majid, S.: Bar categories and star operations. Algebr. Represent. Theory 12, 103-152 (2009)

8. Blackwell, R., Kelly, G.M., Power, A.J.: Two-dimensional monad theory. J. Pure Appl. Algebra 59, 1-41 (1989)

9. Blute, R., Panangaden, P.: Dagger categories and formal distributions. In: Coecke, B. (ed.) New Structures in Physics. Lect. Notes Physics, vol. 813, pp. 421-436. Springer, Berlin (2010)

10. Borceux, F.: Handbook of Categorical Algebra. Encyclopedia of Mathematics, vols. 50-52. Cambridge Univ. Press, Cambridge (1994)

11. Coumans, D., Jacobs, B.: Scalars, monads and categories (2010). arXiv:1003.0585

12. Dvurečenskij, A., Pulmannová, S.: New Trends in Quantum Structures. Kluwer Acad. Publ., Dordrecht (2000)

13. Egger, J.M.: Involutive monoidal categories (2010). Available via http://homepages.inf.ed.ac.uk/ jegger

14. Hasuo, I.: Tracing anonymity with coalgebras. PhD thesis, Radboud Univ. Nijmegen (2010)

15. Hasuo, I., Heunen, C., Jacobs, B., Sokolova, A.: Coalgebraic components in a many-sorted microcosm. In: Kurz, A., Tarlecki, A. (eds.) Conference on Algebra and Coalgebra in Computer Science (CALCO 2009). Lect. Notes Comp. Sci., vol. 5728, pp. 64-80. Springer, Berlin (2009) 
16. Hasuo, I., Jacobs, B., Sokolova, A.: The microcosm principle and concurrency in coalgebra. In: Amadio, R. (ed.) Foundations of Software Science and Computation Structures. LNCS, vol. 4962, pp. 246-260. Springer, Berlin (2008)

17. Jacobs, B.: Categorical Logic and Type Theory. North Holland, Amsterdam (1999)

18. Jacobs, B.: Coalgebraic walks in quantum and Turing computation. In: Hofmann, M. (ed.) Foundations of Software Science and Computation Structures. Lect. Notes Comp. Sci., vol. 6604, pp. 12-26. Springer, Berlin (2011)

19. Jacobs, B.: Dagger categories of tame relations (2011). arXiv:1101.1077

20. Kalmbach, G.: Orthomodular Lattices. Academic Press, London (1983)

21. Kock, A.: Bilinearity and cartesian closed monads. Math. Scand. 29, 161-174 (1971)

22. Kock, A.: Closed categories generated by commutative monads. J. Aust. Math. Soc. XII, 405-424 (1971)

23. Manes, E.G.: Algebraic Theories. Springer, Berlin (1974)

24. Mac Lane, S.: Categories for the Working Mathematician. Springer, Berlin (1971)

25. Street, R.: The formal theory of monads. J. Pure Appl. Algebra 2, 149-169 (1972) 